Int. J. Mol. Sci. 2002, 3, 445-446

International Journal of

Molecular Sciences

ISSN 1422-0067

(C) 2002 by MDPI

www.mdpi.org/ijms/

\title{
Editorial
}

\section{Special Issue on Recent Advances in Coupled Cluster Theory}

\section{Sourav Pal}

Theoretical Chemistry Group, Physical Chemistry Division, National Chemical Laboratory, Pune 411 008, India. E-mail: pal@ems.ncl.res.in

Received: 15 April 2002 / Published: 31 May 2002

The Coupled-cluster (CC) theory has emerged as the most accurate method to describe the electron correlation problem and thus the state-of-the-art electronic structure tool. It is based on exponential ansatz and has correct scaling property. Thus, the CC theory is ideally suited to extended systems. Several theoretical formulations and computational advances have led to the present state of the $\mathrm{CC}$ theory. The connection of the $\mathrm{CC}$ theory with other many-electron theories has also been studied in depth. In particular, connection with perturbation theory has been an important source of understanding. However, many critical aspects of the theory are still under formulation. In recent decades, the most important developments have taken place in the areas of multi-reference variants of the CC theory. These versions of the theory have helped in the applicability of CC methods to the important and demanding quasi-degenerate situations, namely ionized, excited or electron-attached states or potential energy surfaces. There are several versions of the multi-reference CC theory, providing multiple roots as well as single root of interest, which have merits and demerits depending on the area of application. However, several important theoretical problems are yet to be solved and there are scopes of progress and research. Computational developments, introduction of relativistic effects and incorporation of explicitly correlated functions also constitute challenging areas of research, especially in the context of multi-reference version of the theory. Time-dependent CC formulation has also been an important direction of research in this area. A significant development has been the formulation of techniques of analytic derivatives in the context of CC theory. This enabled the CC theory to be applicable to molecular properties, gradients and hessians. In this context, stationary coupled-cluster theory has also been of recent interest. The adaptation of analytic derivatives and appropriate forms of stationary theories in the multi-reference structure of the theory would be of obvious research interest.

The frontier research in coupled cluster theory is an ideal topic of a special issue in this journal, devoted to molecules, for which CC theory has been applied most extensively in recent years. Some of the developments highlighted above have been addressed in this special issue. This issue consists of eight articles, written by experts, and focusing on different aspects of the some of the active areas of 
research in $\mathrm{CC}$ theory. I hope the articles cover the frontiers in the respective areas and the issue will help in further development of CC theory.

(C) 2002 by MDPI (http://www.mdpi.org). 